\title{
Preeclampsia and Extracellular Vesicles
}

\author{
Sarwat I. Gilani ${ }^{1,2}$ • Tracey L. Weissgerber ${ }^{1}$ Vesna D. Garovic ${ }^{1}$. \\ Muthuvel Jayachandran ${ }^{2,3}$
}

Published online: 2 September 2016

(C) The Author(s) 2016. This article is published with open access at Springerlink.com

\begin{abstract}
Preeclampsia is a hypertensive pregnancy disorder characterized by development of hypertension and proteinuria after 20 weeks of gestation that remains a leading cause of maternal and neonatal morbidity and mortality. While preeclampsia is believed to result from complex interactions between maternal and placental factors, the proximate pathophysiology of this syndrome remains elusive. Cell-to-cell communication is a critical signaling mechanism for feto-placental development in normal pregnancies. One mechanism of cellular communication relates to activated cell-derived sealed membrane vesicles called extracellular vesicles (EVs). The concentrations and contents of EVs in biological fluids depend upon their cells of origin and the stimuli which trigger their production. Research on EVs in preeclampsia has focused on EVs derived from the maternal vasculature (endothelium, vascular smooth muscle) and blood (erythrocytes, leukocytes, and platelets), as
\end{abstract}

This article is part of the Topical Collection on Preeclampsia

Muthuvel Jayachandran

jaya.m@mayo.edu

Sarwat I. Gilani

gilani.sarwat@mayo.edu

Tracey L. Weissgerber

weissgerber.tracey@mayo.edu

Vesna D. Garovic

garovic.vesna@mayo.edu

1 Department of Internal Medicine, Division of Nephrology and Hypertension, Mayo Clinic, Rochester, MN 55905, USA

2 Department of Surgery, Mayo Clinic, 200 First Street SW, Rochester, MN 55905, USA

3 Department of Physiology and Biomedical Engineering, Mayo Clinic, Rochester, MN 55905, USA well as placental syncytiotrophoblasts. Changes in the concentrations and contents of these EVs may contribute to the pathophysiology of preeclampsia by accentuating the proinflammatory and pro-coagulatory states of pregnancy. This review focuses on possible interactions among placental- and maternal-derived EVs and their contents in the initiation and progression of the pathogenesis of preeclampsia. Understanding the contributions of EVs in the pathogenesis of preeclampsia may facilitate their use as diagnostic and prognostic biomarkers.

Keywords Hypertensive pregnancy disorder .

Microvesicles · Exosomes · Cell-cell communication . Vesicles

\section{Introduction}

Preeclampsia is characterized by new-onset hypertension (systolic blood pressure $\geq 140 \mathrm{mmHg} /$ diastolic blood pressure $\geq 90 \mathrm{mmHg}$ ), with either proteinuria $(\geq 300 \mathrm{mg} / 24 \mathrm{~h})$ and/or organ dysfunction after 20 weeks of gestation [1]. The underlying cellular and molecular mechanisms that trigger preeclampsia and facilitate its progression are not well understood. Consequently, there are no established early diagnostic tests or effective targeted pharmacological treatments for preeclampsia. The only treatment option is delivery. With a global prevalence rate of $2.7-8.2 \%$ of pregnancies, preeclampsia remains a major challenge in patient management for physicians [2-4].

It is recognized increasingly that preeclampsia is a heterogeneous disease, caused by several distinct underlying mechanisms that may result in different clinical phenotypes [5••]. This is reflected in current clinical practice, as it is common to divide preeclampsia into early ( $<34$ weeks of gestation) and late ( $>34$ weeks of gestation) preeclampsia based on the 
timing of the onset of symptoms. Similarly, preeclampsia may be classified as mild or severe depending on the severity of symptoms, including blood pressure (mild, $<160$ / $110 \mathrm{mmHg}$; severe, $\geq 160 / 110 \mathrm{mmHg}$ ), and the presence or absence of organ dysfunction (kidney failure, liver rupture, stroke, and seizure). Studies investigating the etiologies of preeclampsia have hypothesized that this syndrome may have placental and maternal forms [6]. This approach takes into account the underlying mechanisms. It has been proposed that defects in remodeling of the maternal spiral arteries that supply the placenta ultimately lead to placental ischemia [7••], ischemia reperfusion injury [8], or high velocity blood flow injury in the intervillous space $[9 \cdot \bullet]$ in placental preeclampsia. This triggers the release of one or more placental factors that cause systemic endothelial dysfunction in the maternal circulation. Alternatively, maternal preeclampsia may arise in the setting of vascular dysfunction, oxidative stress, and metabolic abnormalities, such as hypertension, obesity, or diabetes that predate or are exacerbated by pregnancy (in the text that follows, we will refer to these conditions as preeclampsia risk factors). Endothelial dysfunction worsens with advancing gestation as the mother is unable to adapt to the physiological stress of pregnancy (Fig. 1). Placental preeclampsia is commonly viewed as early or severe, while maternal preeclampsia is sometimes characterized as late or mild. Although the dichotomous view of preeclampsia is overly simplistic, the relative contributions of maternal vs. placental factors likely differ among individual women, ultimately resulting in a diverse spectrum of clinical presentations. Irrespective of the predominant underlying mechanism, the interactions among maternal and placental pathophysiological factors may lead to a vicious cycle of maternal inflammation, vascular dysfunction, and the activation of pro- coagulation pathways that ultimately cause the symptoms and signs of preeclampsia.

Based on the complex nature of the origin of preeclampsia, we hypothesize that placental and maternal cells cross-talk, mediated by extracellular vesicles (EVs), contributes to the initiation and progression of preeclampsia in women, both with and without known pre-existing risk factors (Fig. 1). In women for whom EVs derived from the placenta are the major contributors, we propose that the symptoms of preeclampsia may appear earlier in gestation. If EVs derived from maternal cells are the major contributors, the symptoms may appear later in gestation. Two distinct types of EVs (exosomes and microvesicles) are released by almost all activated cells or cells involved in pathophysiological processes [10-13]. Exosomes and microvesicles differ in size and their modes of formation. Exosomes are smaller than microvesicles (30-120 nm vs. $40-1000 \mathrm{~nm}$ ) [12], and are formed by the endocytosis of multivesicular bodies and are released from cells by exocytosis. In contrast, microvesicles (MVs) are membranebound vesicles that are shed from the plasma membrane [12]. Despite these differences, the size ranges for these two distinct classes of EVs overlap in some extent, and there are currently no established methods available to distinguish them purely on basis of size. Surface-specific EV markers that can be used to differentiate microvesicles and exosomes have not yet been identified. We have therefore used the term EVs, as previously suggested by the scientific community [14], to refer to exosomes and microvesicles in this review.

As the role of EVs in the pathophysiology of preeclampsia is an emerging field, the literature contains conflicting data. This review focuses on the most consistent findings, while providing an overview of areas with disparate findings.
Fig. 1 Role of EVs in pathogenesis of preeclampsia. Maternal risk factors and placental abnormalities cause systemic maternal cell activation resulting in release of EVs. Endothelial-, leukocyte-, and platelet-derived EVs give rise to vascular dysfunction, immune modulation, and increased thrombotic propensity. These processes collectively contribute to progression of pathogenesis of preeclampsia

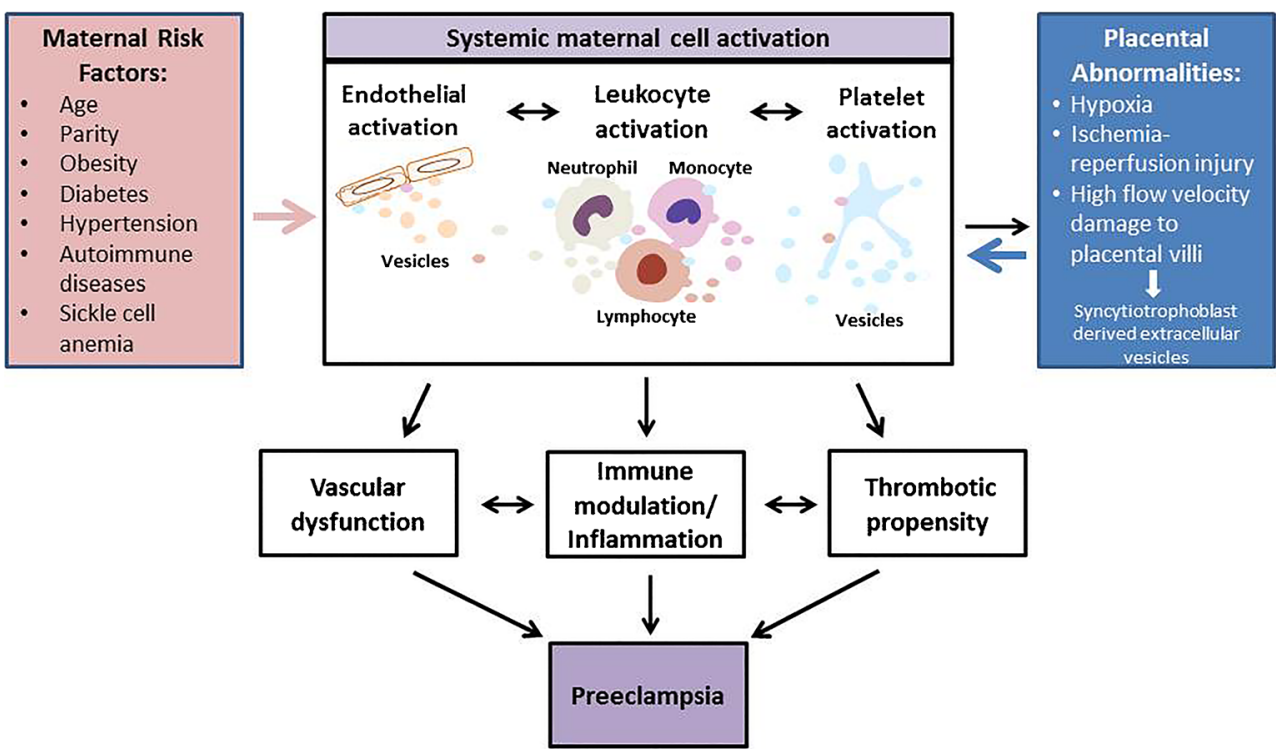




\section{Maternal Cell-Derived EVs Before Pregnancy}

Changes in circulating EVs offer a unique opportunity to examine how preeclampsia risk factors affect the functions of the parent cells and tissues prior to and during pregnancy. Risk factors for preeclampsia include obesity, pre-gestational diabetes mellitus, hypertension, and systemic lupus erythematosus (Table 1). These risk factors could alter the functioning of different types of maternal cells prior to pregnancy, as demonstrated by changes in the concentrations and bioactive molecular contents of the circulating EVs. Studies examining EVs in non-pregnant women suggest that risk factors for preeclampsia are associated with changes in EVs derived from vascular endothelial cells, leukocytes, and platelets [17-26] (Table 2). As shown in Table 2, compared to non-pregnant women without preeclampsia risk factors, non-pregnant women with these risk factors are reported to have increased endothelial-derived EVs [17-19, 22, 24, 25, 27]. Similarly, non-pregnant women with these risk factors are reported to have increased platelet-derived EVs [17, 20-22, 26]; however, they have either increased or decreased concentrations of leukocyte-derived EVs [20, 23-25].

Studies have shown that the effects of preeclampsiaassociated risk factors are similar to the effects of preeclampsia on endothelial-derived EVs. Women with both preeclampsia-associated risk factors and preeclampsia are reported to have increased concentrations of endothelial-derived EVs when compared to either non-pregnant women without these risk factors and/or normotensive pregnant women, respectively [17-19, 22, 24, 25, 27-29]. However, some studies report no change in endothelial-derived EV concentrations in women with preeclampsia compared to normotensive pregnant women [30-32]. Concentrations of leukocyte-derived EV (LEV) in non-pregnant women with preeclampsiaassociated risk factors are reported to be increased, decreased, or not changed, when compared to non-pregnant women without these risk factors [20, 23-25]. Whereas in women with preeclampsia, LEV concentrations are reported to be increased compared to normotensive pregnant women [28, 33]. The effects of preeclampsia-associated risk factors are reported to be opposite to the effects of preeclampsia on platelet-derived EV (PEV). Increased concentrations of PEV are present in women with preeclampsia-associated maternal risk factors compared to non-pregnant women without these risk factors. However, PEV concentrations are reported to be decreased in women with preeclampsia compared to normotensive pregnant women [17, 20-22, 26].

Use of low-dose aspirin is recommended for women with preeclampsia-associated risk factors to decrease the morbidity and mortality associated with preeclampsia [34]. The beneficial effects of aspirin in those with preeclampsia-associated risk factors may, at least in part, be explained by the effect of aspirin on platelet activity. By inhibiting thromboxane $\mathrm{A}_{2}$ synthesis, aspirin decreases platelet activation and, in turn, likely affects the production of PEV. Understanding the effect of aspirin on PEV production and content in women with preeclampsia-associated risk factors may delineate the mechanistic pathways by which PEV contribute to the pathogenesis of preeclampsia.

\section{Placenta-Derived EVs}

The placenta plays a critical role in the pathophysiology of preeclampsia [35]. Placental trophoblasts are involved in spiral artery remodeling and differentiate into extravillous trophoblasts and villous trophoblasts. The villous trophoblasts fuse to form syncytiotrophoblasts. The extravillous trophoblasts invade the distal portions of the spiral arteries, displacing maternal vascular endothelial and smooth muscle cells $[36,37]$. This process transforms the distal portions of the spiral arteries from narrow vessels into wide, flaccid conduits $[38,39 \bullet]$. The uterine oxygen gradient in early pregnancy favors extravillous trophoblast invasion of the uterine spiral arteries and spiral artery remodeling [40]. It is speculated that placental trophoblast-derived EVs $(40-300 \mathrm{~nm})$ [41] may also
Table 1 Preeclampsia-associated maternal risk factors

\begin{tabular}{lll}
\hline Pre-pregnancy maternal characteristics & Relative risk & Reference \\
\hline Obesity & 2.47 & Duckitt et al. [15] \\
Pre-gestational diabetes mellitus & 3.56 & Duckitt et al. [15] \\
Hypertension & $1.38-2.37$ & Duckitt et al. [15] \\
Autoimmune diseases & & \\
• Systemic lupus erythematosus & - & \\
• Antiphospholipid syndrome & 9.72 & Duckitt et al. [15] \\
Sickle cell anemia & 2.43 & Oteng-Ntim et al. [16] \\
Nulliparity & 2.91 & Duckitt et al. [15] \\
Preeclampsia in prior pregnancy & 7.19 & Duckitt et al. [15] \\
Family history of preeclampsia & 2.90 & Duckitt et al. [15] \\
\hline
\end{tabular}


Table 2 EVs in non-pregnant women with preeclampsiaassociated risk factors compared to non-pregnant women without preeclampsia-associated risk factors

\begin{tabular}{|c|c|c|c|}
\hline Risk factor & Extracellular vesicles & Results & Reference \\
\hline \multirow[t]{2}{*}{ Obesity } & Endothelial-derived & $\uparrow$ & Stephanian et al. [17] \\
\hline & Platelet-derived & $\uparrow$ & Stephanian et al. [17] \\
\hline \multirow[t]{5}{*}{ Diabetes mellitus } & Endothelium-derived & $\uparrow$ & Sabatier et al. [18] \\
\hline & & $\uparrow$ & Tramontano et al. [19] \\
\hline & Leukocyte-derived & No difference & Zhang et al. [20] \\
\hline & Platelet-derived & $\uparrow$ & Zhang et al. [20] \\
\hline & & $\uparrow$ & Strano et al. [21] \\
\hline \multirow[t]{2}{*}{ Hypertension } & Endothelium-derived & $\uparrow$ & Preston et al. [22] \\
\hline & Platelet-derived & $\uparrow$ & Preston et al. [22] \\
\hline \multirow[t]{5}{*}{ Systemic lupus erythematosus } & Leukocyte-derived & $\uparrow$ & Lacroix et al. [27] \\
\hline & & $\downarrow$ & Neilson et al. [24] \\
\hline & Endothelium-derived & $\uparrow$ & Lacroix et al. [27] \\
\hline & & No difference & Neilson et al. [24] \\
\hline & Platelet-derived & $\uparrow$ & Stephanian et al. [17] \\
\hline \multirow[t]{2}{*}{ Antiphospholipid syndrome } & Endothelium-derived & $\uparrow$ & Dignat-George et al. [25] \\
\hline & Leukocyte-derived & $\uparrow$ & Dignat-George et al. [25] \\
\hline Sickle cell anemia & Platelet-derived & $\uparrow$ & Wun et al. [26] \\
\hline
\end{tabular}

have a role in spiral artery remodeling [42]. Salomon et al. have shown that oxygen tension regulates the number and protein content of exosomes released by the placenta, with greater release of exosomes by placental trophoblasts under hypoxic conditions in vitro [43, 44]. Placental exosomes are reported to contain serine proteases and metalloproteases (MMP), including MMP-12 [44]. It has been hypothesized that MMP-12 secreted by trophoblasts may facilitate trophoblast invasion by contributing to the remodeling of the extracellular matrix in the vascular wall [45].

Abnormal placentation in women with preeclampsia may increase circulating concentrations of placentalderived EVs. Studies have shown higher concentrations of syncytiotrophoblast- derived EVs [28, 46, 47•, 48], with altered lipid and protein content $[49,50]$, in women with early-onset or severe preeclampsia compared to normotensive pregnant women. In addition, studies suggest that the syncytiotrophoblast apoptosis rate is elevated in preeclampsia (5-6\%) when compared to normal pregnancy $(2-3 \%)$ [51]. In accordance with this finding, higher circulating concentrations of syncytiotrophoblast-derived EVs have been reported in preeclamptic women compared to normotensive pregnant women [41, 46, 47•]. Furthermore, women with early-onset preeclampsia seem to have higher syncytiotrophoblast-derived EVs concentrations than women with late-onset preeclampsia [28, 48, 52•]. Further studies are needed to identify the exact cellular or molecular pathways that stimulate production of placental-derived EVs, which may contribute to the development of preeclampsia.

\section{Effect of Maternal EVs on Production of Placental-Derived EVs}

Dynamic interactions between maternal and fetal factors are constantly occurring at the maternal-fetal interface. These interactions contribute to the regulation of trophoblast phenotype and endovascular invasion. Chemokines and their receptors (CXCR4, CXCR7, CXCL12) promote cell survival and proliferation and inhibit apoptosis [53]. $\mathrm{Lu}$ et al. [53] have shown decreases in the expressions of CXCR4, CXCR7, and CXCL12 molecules in trophoblast cells obtained from the placentas of preeclamptic women. The causes of these decreases are not known. One possible mechanism may be that circulating maternal factors downregulate chemokine receptors at the post-transcriptional level. Alternatively, downregulation of molecules or upregulation of molecular inhibitors at the transcriptional level can also occur in trophoblasts, as demonstrated by Zhou et al. [54]. This study also observed an upregulation of the angiogenesis inhibitor SEMA3B in trophoblasts obtained from women with preeclampsia. SEMA3B inhibits trophoblast invasion of vessels by promoting trophoblast apoptosis. It additionally showed that the increased levels of SEMA3B from preeclamptic trophoblasts returned to control levels after $48 \mathrm{~h}$ in a culture system. Based on this finding, the authors proposed that factors in the maternal milieu cause reversible upregulation of SEMA3B in trophoblasts in preeclampsia [54]. The role of maternal cellderived EVs in regulating trophoblast gene expression in preeclampsia remains to be determined. Holder et al. [55] 
have shown that placental trophoblasts take up exosomes from maternal macrophages and alter the placental production of inflammatory cytokines. Given this evidence, we hypothesize that EVs derived from maternal cells have the potential to alter trophoblast gene expression and function. These changes may contribute to defective trophoblast invasion and increased trophoblast apoptosis.

Placental debris is cleared by macrophages at the maternal-fetal interface [56]. It is known that syncytiotrophoblast-derived vesicles affect the functions of maternal cells, including platelets, leukocytes, erythrocytes, and endothelial cells [57•, 58-62]. Therefore, it is possible that EVs produced by syncytiotrophoblasts also contribute to regulating maternal macrophage activity at the maternal-fetal interface. Exploring the effects of syncytiotrophoblast-derived EVs on maternal macrophages will elucidate the mechanisms by which macrophage activity is regulated at the maternal-fetal interface. The role of maternal EVs in the regulation of trophoblast turnover and immune activity at the maternal-fetal interface will provide valuable insight into the factors regulating the dynamics of the maternal-fetal interface. This, in turn, will delineate the role that EVs of maternal and placental origins and their interactions have in the initiation and progression of placental preeclampsia.

\section{Maternal Cell-Derived EVs and Their Interactions}

Platelet-Derived EVs Platelets are the largest source of EVs in blood in healthy non-pregnant women [63]. When compared to non-pregnant women, most studies report lower platelet-derived $\mathrm{EV}(\mathrm{PEV})$ concentrations in normotensive pregnant women [28, 64]. While most studies have observed further reductions in PEV in women with preeclampsia [28, $31,32,65]$, a few studies have shown higher concentrations of PEV, or no change $[66,67]$ (Table 3). It is plausible that differences in the reported concentrations of PEV among the studies are reflective of different preeclampsia subtypes and their underlying mechanisms. Three different theories have been proposed to explain the lower concentrations of PEV with preeclampsia. (1) Lower platelet counts in preeclampsia may contribute to lower concentrations of PEV [65]. (2) Some studies have hypothesized that the lower concentrations of PEV may partly be due to increased trapping or participation of PEVs in thrombin generation and fibrin clot formation [28, $64,67,72]$. (3) It is postulated that lower PEVs may be due to their association or binding with leukocytes [65].

Leukocyte-Derived EV Studies have shown that leukocyte counts and concentrations of leukocyte-derived EV (LEV) are higher in normotensive pregnant women compared to non-

Table 3 Comparison of EVs between preeclampsia and normotensive pregnancy

\begin{tabular}{|c|c|c|c|}
\hline Parameter & Preeclampsia & $\begin{array}{l}\text { Normotensive } \\
\text { pregnancy }\end{array}$ & Reference \\
\hline Total EVs & $\begin{array}{l}\text { Increased/no } \\
\text { change/decreased }\end{array}$ & Present & $\begin{array}{l}\text { Tesse et al. [68]; Marques et al. [28]; } \\
\text { Mikhailova et al. [33]; VanWijk et al. [69]; } \\
\text { Holthe et al. [70]; Bretelle et al. [32]; } \\
\text { Lok et al. [31] }\end{array}$ \\
\hline $\begin{array}{l}\text { Syncytiotrophoblast-derived } \\
\text { EVs (early-onset PE) }\end{array}$ & $\begin{array}{l}\text { Increased/no } \\
\text { significant change }\end{array}$ & Present & $\begin{array}{l}\text { Knight et al. [46]; Germain et al. [47•]; } \\
\text { Goswami et al. [48]; Lok et al. [31] }\end{array}$ \\
\hline $\begin{array}{l}\text { Syncytiotrophoblast-derived } \\
\text { EVs (late-onset PE)/severe }\end{array}$ & No change/no change & Present & Goswami et al. [48]; [28] \\
\hline Endothelial cell-derived EVs & Increased/no change & Present & $\begin{array}{l}\text { Marques et al. [28]; Gonzalez et al. [29]; } \\
\text { VanWijk et al. [69]; Bretelle et al. [32]; } \\
\text { Lok et al. [31] }\end{array}$ \\
\hline Platelet-derived EVs & Decreased & Present & $\begin{array}{l}\text { Marques et al. [28]; Bretelle et al.[32]; } \\
\text { Lok et al.[28, 31]; Lok et al. [65] }\end{array}$ \\
\hline Leukocyte-derived EVs & Increased & Present & Mikhailova et al. [33]; Marques et al. [28] \\
\hline - Granulocyte-derived EVs & Increased & Present & $\begin{array}{l}\text { Lok et al. [71]; vanWijk et al. [69]; } \\
\text { Marques et al. [28] }\end{array}$ \\
\hline - Monocyte-derived EVs & Increased & Present & Lok et al. [31, 71]; Marques et al.[28] \\
\hline - Lymphocyte-derived EVs & Decreased & Present & Lok et al. [71]; Marques et al.[28] \\
\hline - $\mathrm{T}$ cell-derived EVs & Increased & Negligible & vanWijk et al.[69] \\
\hline - T helper cell-derived EVs & Increased & Negligible & Lok et al. [31] \\
\hline Erythrocyte-derived EVs & Increased & Present & $\begin{array}{l}\text { Lok et al. [31]; Marques et al. [28]; } \\
\text { Dragovic et al. [64] }\end{array}$ \\
\hline
\end{tabular}


pregnant women $[28,33,64,69,71,73]$. The inflammatory state of normotensive pregnancy is further exacerbated in preeclampsia, as preeclamptic women have even higher leukocyte counts and concentrations of LEV [64, 71] (Table 3). The upregulation of granulocytes (neutrophils)-and monocyte- and granulocyte-derived EVs-has been suggested $[28,33,71]$ to aid in the removal and regulation of syncytiotrophoblast-derived vesicles and placental debris that are released into the maternal circulation. Pro-inflammatory EVs can be produced by endothelial cells in response to inflammatory stimuli [74]. Therefore, we speculate that the pro-inflammatory state in preeclampsia may increase circulating concentrations of endothelial-derived pro-inflammatory EVs. These proinflammatory EVs may contribute to the increases in the peripheral blood neutrophils and monocytes in preeclampsia, facilitating the immunomodulation and upregulation of phagocytosis.

The role of EVs in leukocyte activation has also been explored. Studies have shown that syncytiotrophoblast-derived vesicles from normal pregnancy and preeclampsia cause leukocyte activation in vitro $[47 \cdot, 75]$ that is mediated by toll-like receptors and nuclear factor (NF-K $\beta$ ) [76]. Peripheral blood EVs have also been shown to alter monocyte phenotype in vitro [60]. Furthermore, it has been shown that activated leukocytes produce inflammatory cytokines (IL-1, IL-8) and nuclear factor (NF-K $\beta$ ) that can stimulate EV production [77]. The circulating EVs of maternal and placental origins most likely propagate inflammation in preeclampsia [37].

Red Blood Cells (Erythrocytes)-Derived EV While most studies show an increase in the concentrations of red blood cells (erythrocytes)-derived EV (REV) in normotensive pregnant women and preeclamptic women compared to nonpregnant women [28, 31, 64], one study showed no difference [64]. Increased REV concentrations in pregnancy suggest erythrocyte activation, which may be due to increased oxygen demand in pregnancy or stimulation by circulating EVs. Alternatively, increased REV concentrations in preeclampsia may be due to erythrocyte disruption and hemolysis [78], which may be associated with widespread thrombosis [28]. Ten to twenty percent of women with preeclampsia develop hemolysis, elevated liver enzymes, and low platelets (HELLP) syndrome, which is characterized by erythrocyte disruption [79]. Hemolysis, in addition, may result from an autoimmune reaction to trophoblast-derived vesicles deposition on erythrocytes that may explain the increased concentrations of REV in preeclampsia. Determination of REV concentrations and composition in pregnant women with and without preeclampsia and with HELLP syndrome may help to elucidate the mechanisms by which preeclampsia progresses to HELLP syndrome.
Vascular Endothelium and Smooth Muscle Cell-Derived EVs The concentrations of endothelial-derived EV (EEV) are lower in normotensive pregnant women compared to non-pregnant women, reflecting either decreased production of EEV in normotensive pregnancy or EEV binding to circulating blood cells (platelets and leukocytes). Decreased EEV production is associated with decreased peripheral vascular resistance in normotensive pregnancy [80, 81]. Estrogen and maternal fluid dynamics [82] have been postulated to decrease EEV production in normal pregnancy. As syncytiotrophoblast-derived vesicles carry bioactive molecules (e.g., mRNA or miRNA), it may be possible that they regulate the endothelial functions associated with decreased peripheral vascular resistance. Petrozella et al. [83] and VanWijk et al. [69] have shown that circulating EEV concentrations were higher in preeclamptic women compared to normotensive pregnant women (Table 3). This indicates activation of endothelial cells in preeclampsia and an association between increased concentrations of EEV and vascular dysfunction. It has been demonstrated that EEV have differing characteristics depending on signaling stimulus and associated thrombotic and inflammatory processes or conditions [84]. In addition to negatively charged phospholipids, the surface of EEV display receptors (E-selectin, intercellular adhesion molecule-1, and vascular cellular adhesion molecule-1) and markers expressed by endothelial cells. Determining the composition and surface expressions of EEV in normotensive pregnant and preeclamptic women may elucidate the interactions that result in the exacerbation of inflammation and coagulation activation in preeclampsia. EEVs are implicated in the progression of inflammatory vascular diseases [85]. EEV-mediated communication between endothelial cells and the target cells (leukocytes, platelets) is vital to the understanding of the exaggerated pro-inflammatory and pro-coagulation states underlying preeclampsia. Vascular smooth muscle cellderived EVs (SMCEVs) have also been implicated in pathological processes resulting in vascular disease progression [86]. The contributions of SMCEVs in the pathophysiology of preeclampsia have not been investigated. Studies exploring the role of SMCEVs may delineate additional mechanistic vascular pathways contributing to the initiation and progression of preeclampsia.

\section{EVs and Coagulation in Preeclampsia}

The pro-coagulation state of normotensive pregnancy is associated with a decrease in fibrinolytic activity caused by increased pro-coagulant factors and fibrinolytic inhibitors (e.g., plasminogen activator inhibitor-1, [PAI-1]). In addition, the phosphatidylserine present on the surfaces of placental and 
maternal cell-derived EVs contributes to the hypercoagulable state. Thrombin generation and prothrombin fragments increase in normotensive pregnancy [87]. This hypercoagulable state is exaggerated in preeclampsia, resulting in widespread blood clot formation [88], with fibrin deposition in the maternal vasculature, organs, and the placenta [89]. This may be due to the increased total concentrations of pro-coagulant surface positive EVs; however, certain studies have shown no change or even decreases in total counts of MVs in preeclampsia. Alternatively, changes in the phenotype of EVs could also cause increased coagulation. Certain pro-coagulatory molecules (e.g., PAI-1) also have important roles in EEV generation $[90,91]$. Studies exploring the link between pro-coagulant factors and $\mathrm{EV}$ generation in preeclampsia may elucidate the mechanism that links endothelial dysfunction with widespread coagulation. Depending upon the severity of preeclampsia, widespread coagulation activation and clot formation results in ischemic damage in end organs, as well as widespread disseminated intravascular coagulation.

Tissue factor is a ubiquitous $47 \mathrm{kDa}$ transmembrane protein that initiates the inflammatory coagulation pathway. It is present on cells, as well as on cell-derived EVs. It is constitutively expressed in some cells (perivascular fibroblasts) and conditionally expressed in other cells in response to a variety of stimuli, including activated monocytes, macrophages, and the vascular endothelium [92]. The EVs released from activated leukocytes and endothelial cells also express tissue factor [63]. Previous studies have demonstrated that tissue factor is present on the surface of syncytiotrophoblast-derived vesicles $[93,94]$. Upregulation of tissue factor on syncytiotrophoblasts occurs in preeclampsia [95], which is associated with the increased activity of tissue factor in preeclampsia [96]. Gardiner et al. [96] have demonstrated higher tissue factor activity and thrombin generation associated with syncytiotrophoblastderived vesicles from preeclamptic women compared to normotensive pregnant women. Preclinical studies have revealed improved clinical outcomes following anticoagulant therapies in animal models of preeclampsia [97]. Human studies that explore the use of anticoagulants suitable for preeclamptic women are needed. Based on animal studies, anticoagulant therapy has the potential to improve maternal and fetal outcomes in preeclamptic pregnancies.

\section{Conclusions}

EVs have a dynamic role in the communication among maternal vascular cells (the vascular endothelium, circulating leukocytes, and platelets) and the placenta, thus contributing to the progression of normal pregnancy. Depending on pre-existing maternal conditions, any of these vascular components during pregnancy may be capable of initiating the cascade of events that result in preeclampsia. In maternal conditions associated with the activation of vascular endothelial cells and immune system modulation, EEVs can augment inflammation, coagulation, and endothelial dysfunction. Pre-pregnancy maternal platelet activation can augment endothelial dysfunction and inflammation via PEVs, facilitating the progression to preeclampsia. In women without maternal risk factors associated with preeclampsia, it is possible that placental trophoblast-derived EVs may contribute to the maternal milieu that favors progression to preeclampsia.

Future Directions The complex interactions of maternal cell-derived EVs and placental-derived EVs need to be explored further to elucidate the mechanisms of initiation and progression of preeclampsia, with and without known maternal risk factors. In addition to quantitative alterations in EVs, characterizing the bioactive molecular contents (mRNA, miRNA, proteins, lipids, and metabolites) of these EVs based on their cellular origins and their interactions with target cells in the blood and vascular compartments may help to identify underlying mechanisms that contribute to the pathophysiology of preeclampsia. Furthermore, understanding the roles of the specific types of EVs in the pathogenesis of preeclampsia may enable the development of a panel of biomarkers that will help to identify pregnant women at risk for developing preeclampsia. In addition, as maternal vascular, immune, and coagulation systems may have EV-mediated bidirectional communication, it may be possible to therapeutically target one component of the maternal system, which may facilitate the other two systems to return to normalcy in preeclampsia. As most EVs in the healthy pregnant state are derived from platelets, therapeutic interventions aimed at stabilizing platelets can correct the exaggerated pro-inflammatory and hypercoagulable state in preeclampsia that may also help to mitigate the vascular dysfunction and immune flare.

Acknowledgments This study was supported by award number P50-AG44170 (V.D.G. and M.J.) from the National Institute on Aging; by the Building Interdisciplinary Careers in Women's Health award K12HD065987 (T.L.W.) from the Office of Women's Health Research.

\section{Compliance with Ethical Standards}

Conflict of Interest Drs. Gilani, Weissgerber, Garovic, and Jayachandran declare no conflicts of interest relevant to this manuscript.

Human and Animal Rights and Informed Consent This article does not contain any studies with human or animal subjects performed by any of the authors. 
Open Access This article is distributed under the terms of the Creative Commons Attribution 4.0 International License (http:// creativecommons.org/licenses/by/4.0/), which permits unrestricted use, distribution, and reproduction in any medium, provided you give appropriate credit to the original author(s) and the source, provide a link to the Creative Commons license, and indicate if changes were made.

\section{References}

Recently published papers of particular interest have been highlighted:

- of importance

•• of major importance

1. Roberts JM, August PA, Bakris G, Barton JR, Bernstein IM, Druzin $\mathrm{M}$ et al. Hypertension in pregnancy. Report of the American College of Obstetricians and Gynecologists' Task Force on Hypertension in Pregnancy. Obstet Gynecol. 2013;122(5):112231. doi:10.1097/01.AOG.0000437382.03963.88.

2. Abalos E, Cuesta C, Grosso AL, Chou D, Say L. Global and regional estimates of preeclampsia and eclampsia: a systematic review. Eur J Obstet Gynecol Reprod Biol. 2013;170(1):1-7. doi:10.1016/j.ejogrb.2013.05.005.

3. Ananth CV, Keyes KM, Wapner RJ. Pre-eclampsia rates in the United States, 1980-2010: age-period-cohort analysis. BMJ. 2013;347:f6564. doi:10.1136/bmj.f6564.

4. Xiong X, Demianczuk NN, Saunders LD, Wang FL, Fraser WD. Impact of preeclampsia and gestational hypertension on birth weight by gestational age. Am J Epidemiol. 2002;155(3): 203-9.

5.• Myatt L, Redman CW, Staff AC, Hansson S, Wilson ML, Laivuori $\mathrm{H}$, et al. Strategy for standardization of preeclampsia research study design. Hypertension. 2014;63(6):1293-301. doi:10.1161/HYPERTENSIONAHA.113.02664. This article describes methods to standardize the types of preeclampisa for research.

6. Lisonkova S, Joseph KS. Incidence of preeclampsia: risk factors and outcomes associated with early- versus late-onset disease. Am J Obstet Gynecol. 2013;209(6):544.e1-e12. doi:10.1016/j. ajog.2013.08.019.

7.• Roberts JM, Lain KY. Recent Insights into the pathogenesis of pre-eclampsia. Placenta. 2002;23(5):359-72. doi:10.1053/plac.2002.0819. This article describes the properties and functions of extracellular vesicles.

8. Jauniaux E, Hempstock J, Greenwold N, Burton GJ. Trophoblastic oxidative stress in relation to temporal and regional differences in maternal placental blood flow in normal and abnormal early pregnancies. Am J Pathol. 2003;162(1):115-25. doi:10.1016/S00029440(10)63803-5.

9.• Burton GJ, Woods AW, Jauniaux E, Kingdom JC. Rheological and physiological consequences of conversion of the maternal spiral arteries for uteroplacental blood flow during human pregnancy. Placenta. 2009;30(6):473-82. doi:10.1016/j.placenta.2009.02.009. This article describes the generation, secretion and interactions of extracellular vesicles.

10. Yanez-Mo M, Siljander PR, Andreu Z, Zavec AB, Borras FE, Buzas EI, et al. Biological properties of extracellular vesicles and their physiological functions. J Extracell Vesicles. 2015;4:27066. doi:10.3402/jev.v4.27066.

11. EL Andaloussi S, Mager I, Breakefield XO, Wood MJ. Extracellular vesicles: biology and emerging therapeutic opportunities. Nat Rev Drug Discov. 2013;12(5):347-57. doi:10.1038/nrd3978.

12. Colombo M, Raposo G, Thery C. Biogenesis, secretion, and intercellular interactions of exosomes and other extracellular vesicles. Annu Rev Cell Dev Biol. 2014;30:255-89. doi:10.1146/annurevcellbio-101512-122326.

13. Anderson HC, Mulhall D, Garimella R. Role of extracellular membrane vesicles in the pathogenesis of various diseases, including cancer, renal diseases, atherosclerosis, and arthritis. Lab Investig. 2010;90(11):1549-57. doi:10.1038/labinvest.2010.152.

14. Gould SJ, Raposo G. As we wait: coping with an imperfect nomenclature for extracellular vesicles. J Extracell Vesicles. 2013;2. doi:10.3402/jev.v2i0.20389.

15. Duckitt K, Harrington D. Risk factors for pre-eclampsia at antenatal booking: systematic review of controlled studies. BMJ. 2005;330(7491):565. doi:10.1136/bmj.38380.674340.E0.

16. Oteng-Ntim E, Meeks D, Seed PT, Webster L, Howard J, Doyle P, et al. Adverse maternal and perinatal outcomes in pregnant women with sickle cell disease: systematic review and meta-analysis. Blood. 2015;125(21):3316-25. doi:10.1182/blood-2014-11607317.

17. Stepanian A, Bourguignat L, Hennou S, Coupaye M, Hajage D, Salomon L, et al. Microparticle increase in severe obesity: not related to metabolic syndrome and unchanged after massive weight loss. Obesity (Silver Spring). 2013;21(11):223643. doi:10.1002/oby.20365.

18. Sabatier F, Darmon P, Hugel B, Combes V, Sanmarco M, Velut JG, et al. Type 1 and type 2 diabetic patients display different patterns of cellular microparticles. Diabetes. 2002;51(9):2840-5.

19. Tramontano AF, Lyubarova R, Tsiakos J, Palaia T, Deleon JR, Ragolia L. Circulating endothelial microparticles in diabetes mellitus. Mediat Inflamm. 2010;2010:250476. doi:10.1155/2010/250476.

20. Zhang X, McGeoch SC, Johnstone AM, Holtrop G, Sneddon AA, MacRury SM, et al. Platelet-derived microparticle count and surface molecule expression differ between subjects with and without type 2 diabetes, independently of obesity status. J Thromb Thrombolysis. 2014;37(4):455-63. doi:10.1007/s11239-013-1000-2.

21. Strano A, Davi G, Patrono C. In vivo platelet activation in diabetes mellitus. Semin Thromb Hemost. 1991;17(4):422-5. doi:10.1055/s-2007-1002648.

22. Preston RA, Jy W, Jimenez JJ, Mauro LM, Horstman LL, Valle M, et al. Effects of severe hypertension on endothelial and platelet microparticles. Hypertension. 2003;41(2):211-7.

23. Lacroix R, Dignat-George F. Microparticles as a circulating source of procoagulant and fibrinolytic activities in the circulation. Thromb Res. 2012;129 Suppl 2:S27-9. doi:10.1016/j. thromres.2012.02.025.

24. Nielsen CT, Ostergaard O, Johnsen C, Jacobsen S, Heegaard NH. Distinct features of circulating microparticles and their relationship to clinical manifestations in systemic lupus erythematosus. Arthritis Rheum. 2011;63(10):3067-77. doi:10.1002/art.30499.

25. Dignat-George F, Camoin-Jau L, Sabatier F, Arnoux D, Anfosso F, Bardin N, et al. Endothelial microparticles: a potential contribution to the thrombotic complications of the antiphospholipid syndrome. Thromb Haemost. 2004;91(4): 667-73. doi:10.1267/THRO04040667.

26. Wun T, Paglieroni T, Tablin F, Welborn J, Nelson K, Cheung A. Platelet activation and platelet-erythrocyte aggregates in patients with sickle cell anemia. J Lab Clin Med. 1997;129(5):507-16.

27. Lacroix R, Plawinski L, Robert S, Doeuvre L, Sabatier F. Martinez de Lizarrondo $S$ et al. Leukocyte- and endothelial-derived microparticles: a circulating source for fibrinolysis. Haematologica. 2012;97(12):1864-72. doi:10.3324/haematol.2012.066167. 
28. Marques FK, Campos FM, Filho OA, Carvalho AT, Dusse LM, Gomes KB. Circulating microparticles in severe preeclampsia. Clin Chim Acta. 2012;414:253-8. doi:10.1016/j.cca.2012.09.023.

29. Gonzalez-Quintero VH, Smarkusky LP, Jimenez JJ, Mauro LM, Jy W, Hortsman LL, et al. Elevated plasma endothelial microparticles: preeclampsia versus gestational hypertension. Am J Obstet Gynecol. 2004;191(4):1418-24. doi:10.1016/j.ajog.2004.06.044.

30. Vanwijk MJ, Svedas E, Boer K, Nieuwland R, Vanbavel E, Kublickiene KR. Isolated microparticles, but not whole plasma, from women with preeclampsia impair endothelium-dependent relaxation in isolated myometrial arteries from healthy pregnant women. Am J Obstet Gynecol. 2002;187(6):1686-93.

31. Lok CA, Van Der Post JA, Sargent IL, Hau CM, Sturk A, Boer K, et al. Changes in microparticle numbers and cellular origin during pregnancy and preeclampsia. Hypertens Pregnancy. 2008;27(4): 344-60. doi:10.1080/10641950801955733.

32. Bretelle F, Sabatier F, Desprez D, Camoin L, Grunebaum L, Combes V, et al. Circulating microparticles: a marker of procoagulant state in normal pregnancy and pregnancy complicated by preeclampsia or intrauterine growth restriction. Thromb Haemost. 2003;89(3):486-92. doi:10.1267/THRO03030486.

33. Mikhailova VA, Ovchinnikova OM, Zainulina MS, Sokolov DI, Sel'kov SA. Detection of microparticles of leukocytic origin in the peripheral blood in normal pregnancy and preeclampsia. Bull Exp Biol Med. 2014;157(6):751-6. doi:10.1007/s10517-014-2659-x.

34. LeFevre ML. Low-dose aspirin use for the prevention of morbidity and mortality from preeclampsia: U.S. Preventive Services Task Force recommendation statement. Ann Intern Med. 2014;161(11): 819-26. doi:10.7326/M14-1884.

35. Roberts JM, Escudero C. The placenta in preeclampsia. Pregnancy Hypertens. 2012;2(2):72-83. doi:10.1016/j.preghy.2012.01.001.

36. Meekins JW, Pijnenborg R, Hanssens M, McFadyen IR, van Asshe A. A study of placental bed spiral arteries and trophoblast invasion in normal and severe pre-eclamptic pregnancies. Br J Obstet Gynaecol. 1994;101(8):669-74.

37. Roberts JM, Redman CW. Pre-eclampsia: more than pregnancyinduced hypertension. Lancet. 1993;341(8858):1447-51.

38. Zhou Y, Damsky CH, Fisher SJ. Preeclampsia is associated with failure of human cytotrophoblasts to mimic a vascular adhesion phenotype. One cause of defective endovascular invasion in this syndrome? J Clin Invest. 1997;99(9):2152-64. doi:10.1172/JCI119388.

39. Zhou Y, Damsky CH, Chiu K, Roberts JM, Fisher SJ. Preeclampsia is associated with abnormal expression of adhesion molecules by invasive cytotrophoblasts. J Clin Invest. 1993;91(3):950-60. doi:10.1172/JCI116316. An article that describes the role of trophoblast derived vesicles in remodeling of spiral arteries in normal and pathological conditions.

40. Caniggia I, Winter J, Lye SJ, Post M. Oxygen and placental development during the first trimester: implications for the pathophysiology of pre-eclampsia. Placenta. 2000;21(Suppl A):S25-30.

41. Redman CW, Tannetta DS, Dragovic RA, Gardiner C, Southcombe $\mathrm{JH}$, Collett GP, et al. Review: does size matter? Placental debris and the pathophysiology of pre-eclampsia. Placenta. 2012;33(Suppl): S48-54. doi:10.1016/j.placenta.2011.12.006.

42. Salomon C, Yee SW, Mitchell MD, Rice GE. The possible role of extravillous trophoblast-derived exosomes on the uterine spiral arterial remodeling under both normal and pathological conditions. Biomed Res Int. 2014;2014:693157. doi:10.1155/2014/693157.

43. Salomon C, Ryan J, Sobrevia L, Kobayashi M, Ashman K, Mitchell $\mathrm{M}$, et al. Exosomal signaling during hypoxia mediates microvascular endothelial cell migration and vasculogenesis. PLoS One. 2013;8(7), e68451. doi:10.1371/journal.pone.0068451.

44. Salomon C, Kobayashi M, Ashman K, Sobrevia L, Mitchell MD, Rice GE. Hypoxia-induced changes in the bioactivity of cytotrophoblast-derived exosomes. PLoS One. 2013;8(11), e79636. doi:10.1371/journal.pone.0079636.

45. Harris LK, Smith SD, Keogh RJ, Jones RL, Baker PN, Knofler M, et al. Trophoblast- and vascular smooth muscle cell-derived MMP12 mediates elastolysis during uterine spiral artery remodeling. Am J Pathol. 2010;177(4):2103-15. doi:10.2353/ajpath.2010.100182.

46. Knight M, Redman CW, Linton EA, Sargent IL. Shedding of syncytiotrophoblast microvilli into the maternal circulation in preeclamptic pregnancies. Br J Obstet Gynaecol. 1998;105(6):632-40.

47. Germain SJ, Sacks GP, Sooranna SR, Sargent IL, Redman CW. Systemic inflammatory priming in normal pregnancy and preeclampsia: the role of circulating syncytiotrophoblast microparticles. J Immunol. 2007;178(9):5949-56. An article that describes the proteomic anaylsis of trophoblast derived vesicles.

48. Goswami D, Tannetta DS, Magee LA, Fuchisawa A, Redman CW, Sargent IL, et al. Excess syncytiotrophoblast microparticle shedding is a feature of early-onset pre-eclampsia, but not normotensive intrauterine growth restriction. Placenta. 2006;27(1):56-61. doi:10.1016/j.placenta.2004.11.007.

49. Baig S, Lim JY, Fernandis AZ, Wenk MR, Kale A, Su LL, et al. Lipidomic analysis of human placental syncytiotrophoblast microvesicles in adverse pregnancy outcomes. Placenta. 2013;34(5):436-42. doi:10.1016/j.placenta.2013.02.004.

50. Baig S, Kothandaraman N, Manikandan J, Rong L, Ee KH, Hill J, et al. Proteomic analysis of human placental syncytiotrophoblast microvesicles in preeclampsia. Clin Proteomics. 2014;11(1):40. doi:10.1186/1559-0275-11-40.

51. Leung DN, Smith SC, To KF, Sahota DS, Baker PN. Increased placental apoptosis in pregnancies complicated by preeclampsia. Am J Obstet Gynecol. 2001;184(6):1249-50. doi:10.1067/mob.2001.112906.

52. Chen Y, Huang Y, Jiang R, Teng Y. Syncytiotrophoblast-derived microparticle shedding in early-onset and late-onset severe preeclampsia. Int J Gynaecol Obstet. 2012;119(3):234-8. doi:10.1016/j.ijgo.2012.07.010. This article describes the effect of macrohpage vesicles on production of placental inflammatory cytokines.

53. Lu J, Zhou WH, Ren L, Zhang YZ. CXCR4, CXCR7, and CXCL12 are associated with trophoblastic cells apoptosis and linked to pathophysiology of severe preeclampsia. Exp Mol Pathol. 2015;100(1):184-91. doi:10.1016/j.yexmp.2015.12.013.

54. Zhou Y, Gormley MJ, Hunkapiller NM, Kapidzic M, Stolyarov Y, Feng V, et al. Reversal of gene dysregulation in cultured cytotrophoblasts reveals possible causes of preeclampsia. J Clin Invest. 2013;123(7):2862-72. doi:10.1172/JCI66966.

55. Holder BS, Jones T, Sancho Shimizu V, Rice TF, Donaldson B, Bouqueau M, et al. Macrophage exosomes induce placental inflammatory cytokines: a novel mode of maternal-placental messaging. Traffic. 2015. doi:10.1111/tra.12352.

56. Abrahams VM, Kim YM, Straszewski SL, Romero R, Mor G. Macrophages and apoptotic cell clearance during pregnancy. Am J Reprod Immunol. 2004;51(4):275-82. doi:10.1111/j.16000897.2004.00156.x.

57. Tannetta DS, Hunt K, Jones CI, Davidson N, Coxon CH, Ferguson $\mathrm{D}$, et al. Syncytiotrophoblast extracellular vesicles from preeclampsia placentas differentially affect platelet function. PLoS One. 2015;10(11), e0142538. doi:10.1371/journal.pone.0142538. This article describes the effect of circulatory vesicles on monocytes in preeclampsia.

58. Cockell AP, Learmont JG, Smarason AK, Redman CW, Sargent IL, Poston L. Human placental syncytiotrophoblast microvillous membranes impair maternal vascular endothelial function. Br J Obstet Gynaecol. 1997;104(2):235-40.

59. Messerli M, May K, Hansson SR, Schneider H, Holzgreve W, Hahn $\mathrm{S}$, et al. Feto-maternal interactions in pregnancies: placental 
microparticles activate peripheral blood monocytes. Placenta. 2010;31(2):106-12. doi:10.1016/j.placenta.2009.11.011.

60. Sokolov DI, Ovchinnikova OM, Korenkov DA, Viknyanschuk AN, Benken KA, Onokhin KV, et al. Influence of peripheral blood microparticles of pregnant women with preeclampsia on the phenotype of monocytes. Transl Res. 2014. doi:10.1016/j. trs1.2014.11.009.

61. Southcombe J, Tannetta D, Redman C, Sargent I. The immunomodulatory role of syncytiotrophoblast microvesicles. PLoS One. 2011;6(5), e20245. doi:10.1371/journal.pone.0020245.

62. Tong M, Chamley LW. Placental extracellular vesicles and fetomaternal communication. Cold Spring Harb Perspect Med. 2015;5(3):a023028. doi:10.1101/cshperspect.a023028.

63. Gustafson CM, Shepherd AJ, Miller VM, Jayachandran M. Age- and sex-specific differences in blood-borne microvesicles from apparently healthy humans. Biol Sex Differ. 2015;6:10. doi:10.1186/s13293015-0028-8.

64. Dragovic RA, Southcombe JH, Tannetta DS, Redman CW, Sargent IL. Multicolor flow cytometry and nanoparticle tracking analysis of extracellular vesicles in the plasma of normal pregnant and pre-eclamptic women. Biol Reprod. 2013;89(6): 151. doi:10.1095/biolreprod.113.113266.

65. Lok CA, Nieuwland R, Sturk A, Hau CM, Boer K, Vanbavel E, et al. Microparticle-associated P-selectin reflects platelet activation in preeclampsia. Platelets. 2007;18(1):68-72. doi:10.1080/09537100600864285.

66. Meziani F, Tesse A, David E, Martinez MC, Wangesteen R, Schneider F, et al. Shed membrane particles from preeclamptic women generate vascular wall inflammation and blunt vascular contractility. Am J Pathol. 2006;169(4):1473-83. doi:10.2353/ajpath.2006.051304.

67. Alijotas-Reig J, Palacio-Garcia C, Farran-Codina I, Ruiz-Romance M, Llurba E, Vilardell-Tarres M. Circulating cell-derived microparticles in severe preeclampsia and in fetal growth restriction. Am J Reprod Immunol. 2012;67(2):140-51. doi:10.1111/j.16000897.2011.01072.x.

68. Tesse A, Meziani F, David E, Carusio N, Kremer H, Schneider F, et al. Microparticles from preeclamptic women induce vascular hyporeactivity in vessels from pregnant mice through an overproduction of NO. Am J Physiol Heart Circ Physiol. 2007;293(1): H520-5. doi:10.1152/ajpheart.01094.2006.

69. VanWijk MJ, Nieuwland R, Boer K, van der Post JA, VanBavel E, Sturk A. Microparticle subpopulations are increased in preeclampsia: possible involvement in vascular dysfunction? Am J Obstet Gynecol. 2002;187(2):450-6.

70. Holthe MR, Lyberg T, Staff AC, Berge LN. Leukocyte-platelet interaction in pregnancies complicated with preeclampsia. Platelets. 2005;16(2):91-7. doi:10.1080/09537100400004355.

71. Lok CA, Jebbink J, Nieuwland R, Faas MM, Boer K, Sturk A, et al. Leukocyte activation and circulating leukocyte-derived microparticles in preeclampsia. Am J Reprod Immunol. 2009;61(5):346-59. doi:10.1111/j.1600-0897.2009.00701.x.

72. Campello E, Spiezia L, Radu CM, Dhima S, Visentin S, Valle FD, et al. Circulating microparticles in umbilical cord blood in normal pregnancy and pregnancy with preeclampsia. Thromb Res. 2015. doi:10.1016/j.thromres.2015.05.029.

73. Lurie S, Frenkel E, Tuvbin Y. Comparison of the differential distribution of leukocytes in preeclampsia versus uncomplicated pregnancy. Gynecol Obstet Investig. 1998;45(4):229-31.

74. Maurus CF, Schneider MK, Schmidt D, Zund G, Seebach JD. Activation of human microvascular endothelial cells with TNFalpha and hypoxia/reoxygenation enhances NK-cell adhesion, but not NK-Cytotoxicity. Transplantation. 2006;81(8):1204-11. doi:10.1097/01.tp.0000205175.53938.bd.

75. Holder BS, Tower CL, Jones CJ, Aplin JD, Abrahams VM. Heightened pro-inflammatory effect of preeclamptic placental microvesicles on peripheral blood immune cells in humans. Biol Reprod. 2012;86(4):103. doi:10.1095/biolreprod.111.097014.

76. Joerger-Messerli MS, Hoesli IM, Rusterholz C, Lapaire O. Stimulation of monocytes by placental microparticles involves toll-like receptors and nuclear factor kappa-light-chainenhancer of activated B cells. Front Immunol. 2014;5:173. doi:10.3389/fimmu.2014.00173.

77. Leroyer AS, Anfosso F, Lacroix R, Sabatier F, Simoncini S, Njock SM, et al. Endothelial-derived microparticles: biological conveyors at the crossroad of inflammation, thrombosis and angiogenesis. Thromb Haemost. 2010;104(3):456-63. doi:10.1160/TH10-02-0111.

78. Sarrel PM, Lindsay DC, Poole-Wilson PA, Collins P. Hypothesis: inhibition of endothelium-derived relaxing factor by haemoglobin in the pathogenesis of pre-eclampsia. Lancet. 1990;336(8722):1030-2.

79. Fish R. The HELLP syndrome: case report and review of the literature. J Emerg Med. 1993;11(2):169-74.

80. Deanfield JE, Halcox JP, Rabelink TJ. Endothelial function and dysfunction: testing and clinical relevance. Circulation. 2007;115(10): 1285-95. doi:10.1161/CIRCULATIONAHA.106.652859.

81. Choi JW, Im MW, Pai SH. Nitric oxide production increases during normal pregnancy and decreases in preeclampsia. Ann Clin Lab Sci. 2002;32(3):257-63.

82. Easterling TR, Benedetti TJ, Schmucker BC, Millard SP. Maternal hemodynamics in normal and preeclamptic pregnancies: a longitudinal study. Obstet Gynecol. 1990;76(6):1061-9.

83. Petrozella L, Mahendroo M, Timmons B, Roberts S, McIntire D, Alexander JM. Endothelial microparticles and the antiangiogenic state in preeclampsia and the postpartum period. Am J Obstet Gynecol. 2012;20(2):140.e20-6. doi:10.1016/j.ajog.2012.06.011.

84. Jimenez JJ, Jy W, Mauro LM, Soderland C, Horstman LL, Ahn YS. Endothelial cells release phenotypically and quantitatively distinct microparticles in activation and apoptosis. Thromb Res. 2003;109(4):175-80.

85. Dignat-George $\mathrm{F}$, Boulanger $\mathrm{CM}$. The many faces of endothelial microparticles. Arterioscler Thromb Vasc Biol. 2011;31(1):27-33. doi:10.1161/ATVBAHA.110.218123.

86. Kapustin AN, Shanahan CM. Calcium regulation of vascular smooth muscle cell-derived matrix vesicles. Trends Cardiovasc Med. 2012;22(5):133-7. doi:10.1016/j.tcm.2012.07.009.

87. Rosenkranz A, Hiden M, Leschnik B, Weiss EC, Schlembach D, Lang $\mathrm{U}$, et al. Calibrated automated thrombin generation in normal uncomplicated pregnancy. Thromb Haemost. 2008;99(2):331-7. doi:10.1160/TH07-05-0359.

88. Bonnar J, McNicol GP, Douglas AS. Coagulation and fibrinolytic systems in pre-eclampsia and eclampsia. Br Med J. 1971;2(5752): 12-6.

89. Brown MA. The physiology of pre-eclampsia. Clin Exp Pharmacol Physiol. 1995;22(11):781-91.

90. Reith A, Booth NA, Moore NR, Cruickshank DJ, Bennett B. Plasminogen activator inhibitors (PAI-1 and PAI-2) in normal pregnancies, pre-eclampsia and hydatidiform mole. Br J Obstet Gynaecol. 1993;100(4):370-4.

91. Brodsky SV, Malinowski K, Golightly M, Jesty J, Goligorsky MS. Plasminogen activator inhibitor-1 promotes formation of endothelial microparticles with procoagulant potential. Circulation. 2002;106(18):2372-8.

92. Chu AJ. Tissue factor, blood coagulation, and beyond: an overview. Int J Inflamm. 2011;2011:367284. doi:10.4061/2011/367284.

93. Teng YC, Lin QD, Lin JH, Ding CW, Zuo Y. Coagulation and fibrinolysis related cytokine imbalance in preeclampsia: the role of placental trophoblasts. J Perinat Med. 2009;37(4):343-8. doi:10.1515/JPM.2009.060.

94. Aharon A, Brenner B, Katz T, Miyagi Y, Lanir N. Tissue factor and tissue factor pathway inhibitor levels in trophoblast cells: 
implications for placental hemostasis. Thromb Haemost. 2004;92(4):776-86. doi:10.1267/THRO04040776.

95. Teng Y, Jiang R, Lin Q, Ding C, Ye Z. The relationship between plasma and placental tissue factor, and tissue factor pathway inhibitors in severe pre-eclampsia patients. Thromb Res. 2010;126(1): e41-5. doi:10.1016/j.thromres.2010.02.012.

96. Gardiner C, Tannetta DS, Simms CA, Harrison P, Redman $\mathrm{CW}$, Sargent IL. Syncytiotrophoblast microvesicles released from pre-eclampsia placentae exhibit increased tissue factor activity. PLoS One. 2011;6(10), e26313. doi:10.1371/journal. pone. 0026313 .

97. Zhang Y, Hua Z, Zhang K, Meng K, Hu Y. Therapeutic effects of anticoagulant agents on preeclampsia in a murine model induced by phosphatidylserine/phosphatidylcholine microvesicles. Placenta. 2009;30(12):1065-70. doi:10.1016/j. placenta.2009.09.004. 